\title{
A Note on Hypertension Classification Scheme and Soft Computing Decision Making System
}

\author{
Pankaj Srivastava, ${ }^{1}$ Amit Srivastava, ${ }^{2}$ Anjali Burande, ${ }^{3}$ and Amit Khandelwal ${ }^{2}$ \\ ${ }^{1}$ Department of Mathematics, Motilal Nehru National Institute of Technology, Allahabad 211004, India \\ ${ }^{2}$ Applied Mechanics Department, Motilal Nehru National Institute of Technology, Allahabad 211004, India \\ ${ }^{3}$ Department of Mathematics, Dayanand College of Commerce, Latur 413512, India
}

Correspondence should be addressed to Pankaj Srivastava; pankajs23@rediffmail.com

Received 22 July 2013; Accepted 28 August 2013

Academic Editors: V. P. Emerenciano and J. R. C. Piqueira

Copyright (C) 2013 Pankaj Srivastava et al. This is an open access article distributed under the Creative Commons Attribution License, which permits unrestricted use, distribution, and reproduction in any medium, provided the original work is properly cited.

\begin{abstract}
Nowadays young professionals are a soft target of hypertension due to the increased work pressure and poor tolerance. Many people have high blood pressure for years without knowing it. Most of the time, there are no symptoms, but when this condition goes untreated it damages arteries and vital organs throughout the body and that is why it is also termed as the silent killer. Complications arising from hypertension could lead to stroke and heart failure. Soft computing approach provides a sharper conclusion from vague, ambiguous, and imprecise data (generally found in medical field) using linguistic variables. In this study, a soft computing diagnostic support system for the risk assessment of hypertension is proposed.
\end{abstract}

\section{Introduction}

A human body is a complex system and there are a number of variables that affect its functioning. The abnormality in its functioning causes a number of symptoms in the form of primary stages of different diseases although the recognition of these symptoms and their mapping with the diseases precisely is not an easy one. Sometimes compications in human body may be caused by improper diagnosis or improper management of the disease or due to the inaccessibility of medical personnel [1]. The quickening speed of change and adoption of western lifestyles by people in developing countries have led to a sharp rise in the incidence of hypertension [2]. Hypertension is a medical term for high blood pressure which is a condition that occurs when the pressure in the arteries is above the normal range. According to one of the studies "Recession has had an adverse impact on jobs in India and perhaps this is one of the reasons why cases of Hypertension have gone up in past two years among young IT professionals". Recent analysis has predicted that more than 1.56 billon people will be living with hypertension worldwide by the year 2025. It has been declared by a survey report that one of four adults in India has high BP which kills 7.5 million people worldwide each year; moreover, AIDS, diabetes, road accidents, and tuberculosis are put together. In India $23.1 \%$ men and $22.6 \%$ women have high BP a notch lower than the global prevalence of one in three adults says the World Health statistics 2012 released, 16 May 2012. Jain [3] established a decision making process phenomenon in the presence of fuzzy variables. Poli et al. [4] developed a neural network expert system for diagnosing and treating hypertension. Degani [5] discussed computerized electrocardiogram diagnosis using fuzzy approach. Charbonnier et al. [6] proposed the statistical and fuzzy models of ambulatory systolic blood pressure for hypertension diagnosis. Jena et al. [7] discussed the application of soft computing in medical science. Pandey et al. [8] proposed a rule based system for cardiac analysis based on electrocardiography. Further, Allahverdi et al. [9] proposed a fuzzy expert system for the determination of coronary heart disease risk (CHD) of patient for the next ten years. Nalayini and Wahidabanu [10] were of the view that most of the cardiac diseases are characterized by varied degrees of intricacy and the conventional procedures are not capable of dealing with these intricacies very efficiently. Djam and Kimbi [1] developed a 
TABLE 1

\begin{tabular}{lcc}
\hline & Systolic BP in mm Hg & Diastolic BP in mm Hg \\
\hline Desirable & $90-120$ & $60-80$ \\
Above desirable & $120-130$ & $80-85$ \\
Moderate & $130-140$ & $85-90$ \\
Above moderate & $140-150$ & $90-95$ \\
Little high & $150-160$ & $95-100$ \\
High & $160-170$ & $100-110$ \\
Very high & $>168$ & $>108$ \\
\hline
\end{tabular}

fuzzy expert system for the management of hypertension. Recently, P. Srivastava and A. Srivastava [11] proposed a soft computing diagnostic system to evaluate the risk factor for coronary heart disease (CHD). Srivastava and Sharma [12] designed a soft computing diagnostic system that classifies ECG beats in different phases and enables us to identify the status of cardiac health as per available ECG graphs. The present paper introduces a new soft computing model that measures risk factor on the basis of newly designed algorithm; a number of cases have been discussed as per available database.

\section{Methodology}

For complex systems, fuzzy tools are quite suitable because of its tolerance to some imprecision. In the present study, the inputs consist of age, systolic blood pressure (SBP), diastolic blood pressure (DBP), body mass index (BMI), heart rate, low density lipoprotein (LDL), high density lipoprotein (HDL), triglyceride, smoking, and exercise, while the output is the risk grade of hypertension.

In order to design a user friendly informative system for evaluating risk percentage of hypertension, we propose fuzzy Algorithm 1.

\subsection{Input Variables}

2.1.1. Blood Pressure. In this field we use systolic BP (SBP) and diastolic $\mathrm{BP}$ (DBP). The input variables for SBP and $\mathrm{DBP}$ were classified into seven fuzzy sets (see Table 1).

Systolic Blood Pressure (SBP). Consider

$$
\begin{aligned}
& \mu_{\text {above desirable }}= \begin{cases}0 & x \leq 120 \\
\frac{x-120}{3} & 120<x \leq 123 \\
1 \frac{130-x}{3} & 123<x \leq 127 \\
0 & x \geq 130,\end{cases} \\
& \mu_{\text {moderate }}= \begin{cases}0 & x \leq 130 \\
\frac{x-130}{3} & 130<x \leq 133 \\
1 & 133<x \leq 137 \\
\frac{140-x}{3} & 137<x \leq 140 \\
0 & x \geq 140,\end{cases} \\
& \mu_{\text {above moderate }}= \begin{cases}0 & x \leq 140 \\
\frac{x-140}{3} & 140<x \leq 143 \\
1 & 143<x \leq 147 \\
\frac{150-x}{3} & 147<x \leq 150 \\
0 & x \geq 150,\end{cases} \\
& \mu_{\text {little high }}= \begin{cases}0 & x \leq 150 \\
\frac{x-150}{3} & 150<x \leq 153 \\
1 & 153<x \leq 157 \\
\frac{160-x}{3} & 157<x \leq 160 \\
0 & x \geq 160,\end{cases} \\
& \mu_{\text {high }}= \begin{cases}0 & x \leq 160 \\
\frac{x-160}{3} & 160<x \leq 163 \\
1 & 163<x \leq 167 \\
\frac{170-x}{3} & 167<x<170 \\
0 & x \geq 170\end{cases} \\
& \mu_{\text {very high }}= \begin{cases}0 & x \leq 168 \\
\left(\frac{x-168}{6}\right)^{2} & 168 \leq x<174 \\
1-\left(\frac{x-174}{6}\right)^{2} & 174 \leq x<180 \\
1 & x \geq 180 .\end{cases}
\end{aligned}
$$

Diastolic Blood Pressure (DBP)

$$
\mu_{\text {desirable }}= \begin{cases}1 & x \leq 90 \\ \left(\frac{x-90}{15}\right)^{2} & 90 \leq x<105 \\ 1-\left(\frac{x-105}{15}\right)^{2} & 105 \leq x<120 \\ 0 & x \geq 120,\end{cases}
$$$$
\mu_{\text {normal }}= \begin{cases}1 & x \leq 60 \\ \left(\frac{x-60}{10}\right)^{2} & 60 \leq x<70 \\ 1-\left(\frac{x-70}{10}\right)^{2} & 70 \leq x<80 \\ 0 & x \geq 80,\end{cases}
$$ 
(I) Initial fuzzification mechanism BEGIN

(1) Input-Fuzzy system with suitable " $n$ " parameters $A_{i}$

(2) Initialize $i \leftarrow 1$

DO UNTIL $(i>n)$

Categorize $n_{i}$ fuzzy sets in $X_{j}$ linguistic variables

DO UNTIL $\left(j>n_{i}\right)$

(1) Construction of suitable membership function $\mu_{X}$

(2) Increment $j$

END DO UNTIL

Increment $i$

END

(II) Construction of Fuzzy Strings

\section{BEGIN}

(1) Input: " $n_{i}$ " fuzzy sets in linguistic variables $X_{j} ; i=1,2, \ldots, n$ and $j=1,2, \ldots, n_{i}$

(2) Input: $m$ output parameters $Y_{o}, o=1,2, \ldots, m$.

(3) Develop $t=k_{1}, k_{2}, \ldots, k_{r}$ linguistic strings $J_{k} ; k=1,2, \ldots, t$ using AND operation on each linguistic term $X_{j} ; j=1,2, \ldots, n_{i}$

(4) Initialize $k \leftarrow 1$

DO UNTIL $(k>t)$

Increment $j$

END DO UNTIL

Increment $k$

END

(III) Output Evaluation

\section{BEGIN}

(1) Construct Utility matrix $U$ of pxq order.

(2) Construct $r$ utility fuzzy sets $U_{I} ; I=1,2, \ldots r$ using $x \oplus y=x+y-x y$ for each $x, y \in U$

(3) Initialize $I \leftarrow 1$ DO UNTIL $(I>r)$

(4) Construct $r$ maximizing sets $U_{M I}, I=1,2 \ldots r$ corresponding to each alternative.

(5) Develop $r$ optimal fuzzy utility sets $U_{\mathrm{OI}}, I=1,2 \ldots r$. Each optimal fuzzy set $U_{\mathrm{OI}}$ is obtained by fuzzy intersection $\bigwedge$ on fuzzy utility set and maximizing set such that $\mu_{U_{O I}}(x)=\mu_{U_{I}}(x) \bigcap \mu_{U_{M I}}(x)=\min \left(\mu_{U_{I}}(x), \mu_{U_{M I}}(x)\right)$ for each utility value $x$.

(6) Take maximum membership value from each optimal utility fuzzy set.

(7) The optimal alternative $A_{O}$ with corresponding maximum membership grades obtained in step 10 such as $A_{O}=\left\{\left((\max ) \mu_{U_{O I}}(x), B_{I}\right): \bigvee x \in U_{O I}\right\}$ for $I=1,2 \ldots r$. END DO UNTIL

Increment $I$

END

Algorithm 1

$$
\mu_{\text {above normal }}=\left\{\begin{array}{ll}
0 & x \leq 80 \\
\frac{x-80}{2} & 80<x \leq 82 \\
\frac{85-x}{3} & 82<x \leq 85 \\
0 & x \geq 85,
\end{array} \quad \mu_{\text {moderate }}= \begin{cases}0 & x \leq 85 \\
\frac{x-85}{2} & 85<x \leq 87 \\
\frac{90-x}{3} & 87<x \leq 90 \\
0 & x \geq 90,\end{cases}\right.
$$


TABLE 2

\begin{tabular}{lccc}
\hline & \multicolumn{2}{c}{ Cholesterol $(\mathrm{mg} / \mathrm{dL})$} & HDL \\
\hline LDL & & Very high & $\leq 30$ \\
Above normal & $100-130$ & High & $30-50$ \\
Borderline high & $130-160$ & Nearly normal & $50-60$ \\
High & $160-190$ & Normal & $\geq 58$ \\
Very high & $\geq 180$ & & \\
\hline
\end{tabular}

$$
\begin{gathered}
\mu_{\text {above moderate }}= \begin{cases}0 & x \leq 90 \\
\frac{x-90}{2} & 90<x \leq 92 \\
\frac{95-x}{3} & 92<x \leq 95 \\
0 & x \geq 95,\end{cases} \\
\mu_{\text {little high }}= \begin{cases}0 & x \leq 95 \\
\frac{x-95}{2} & 95<x \leq 97 \\
\frac{100-x}{3} & 97<x \leq 100 \\
0 & x \geq 100,\end{cases} \\
\mu_{\text {high }}= \begin{cases}0 & x \leq 100 \\
\frac{x-100}{5} & 100<x \leq 105 \\
\frac{110-x}{5} & 105<x \leq 110 \\
0 & x \geq 110,\end{cases} \\
\mu_{\text {very high }}= \begin{cases}0 & x \leq 108 \\
\left.\frac{x-108}{6}\right)^{2} & 108 \leq x<114 \\
1-\left(\frac{x-114}{6}\right)^{2} & 114 \leq x<120 \\
1 & x \geq 120 .\end{cases}
\end{gathered}
$$

(2)

2.1.2. Cholesterol. In this study we have classified total cholesterol into low density lipoprotein (LDL) cholesterol and high density lipoprotein (HDL). HDL cholesterol level has been classified into four fuzzy sets. LDL cholesterol level has been classified into five fuzzy sets. High levels of LDL are associated with coronary artery disease, whereas high levels of HDL appear to protect against coronary artery disease. These fuzzy sets have been shown in Table 2.
Low Density Lipoprotein (LDL). Consider

$$
\begin{aligned}
& \mu_{\text {normal }}= \begin{cases}1 & x \leq 50 \\
\left(\frac{x-50}{25}\right)^{2} & 50 \leq x<75 \\
1-\left(\frac{x-75}{25}\right)^{2} & 75 \leq x<100 \\
0 & x \geq 100,\end{cases} \\
& \mu_{\text {above normal }}= \begin{cases}0 & x \leq 100 \\
\frac{x-100}{10} & 100<x \leq 110 \\
1 \frac{130-x}{10} & 110 \leq x \leq 120 \\
0 & x \geq 130,\end{cases} \\
& \mu_{\text {borderline high }}= \begin{cases}0 & x \leq 130 \\
\frac{x-130}{10} & 130<x \leq 140 \\
1 & 140 \leq x \leq 150 \\
\frac{160-x}{10} & 150<x \leq 160 \\
0 & x \geq 160\end{cases} \\
& \mu_{\text {high }}= \begin{cases}0 & x \leq 160 \\
\frac{x-160}{10} & 160<x \leq 170 \\
1 & 170 \leq x \leq 180 \\
\frac{190-x}{10} & 180<x \leq 190 \\
0 & x \geq 190,\end{cases} \\
& \mu_{\text {very high }}= \begin{cases}0 & x \leq 180 \\
\left(\frac{x-190}{20}\right)^{2} & 180 \leq x<200 \\
1-\left(\frac{x-200}{20}\right)^{2} & 200 \leq x<220 \\
1 & x \geq 220 .\end{cases}
\end{aligned}
$$

High Density Lipoprotein (HDL). Consider 


$$
\begin{gathered}
\mu_{\text {high }}= \begin{cases}\frac{x-30}{5} & 30<x \leq 35 \\
1 \frac{50-x}{5} & 35<x \leq 45\end{cases} \\
\mu_{\text {nearly normal }}= \begin{cases}\frac{1}{3} & x \geq 50, \\
\frac{60-x}{3} & 57<x \leq 60 \\
\frac{x-50}{0} & 53<x \leq 53\end{cases} \\
\mu_{\text {normal }}= \begin{cases}0 & x \geq 60, \\
1-\left(\frac{x-65}{5}\right)^{2} & 65 \leq x<70 \\
1 & x \geq 70 .\end{cases}
\end{gathered}
$$

2.1.3. Age. This input field is classified into six fuzzy sets. The fuzzy sets with their range are shown in Table 3. Consider

$$
\begin{aligned}
& \mu_{\text {young }}= \begin{cases}1 & x \leq 0 \\
\left(\frac{x-0}{15}\right)^{2} & 0 \leq x<15 \\
1-\left(\frac{x-15}{15}\right)^{2} & 15 \leq x<30 \\
0 & x \geq 30,\end{cases} \\
& \mu_{\text {adult }}= \begin{cases}0 & x<25 \\
\frac{x-25}{5} & 25 \leq x \leq 30 \\
1 & 30 \leq x \leq 40 \\
\frac{48-x}{8} & 40 \leq x \leq 48 \\
0 & x \geq 48\end{cases} \\
& \mu_{\text {mid aged }}= \begin{cases}0 & x<45 \\
\frac{x-45}{5} & 45 \leq x \leq 50 \\
1 & 50 \leq x \leq 56 \\
\frac{60-x}{4} & 56 \leq x \leq 60 \\
0 & x \geq 60\end{cases} \\
& \mu_{\text {aged }}= \begin{cases}0 & x<58 \\
\frac{x-58}{4} & 58 \leq x \leq 62 \\
1 & 62 \leq x \leq 66 \\
\frac{72-x}{6} & 66 \leq x \leq 72 \\
0 & x \geq 72\end{cases}
\end{aligned}
$$

Table 3

\begin{tabular}{lc}
\hline & Age (in years) \\
\hline Young & $<30$ \\
Adult & $25-48$ \\
Midaged & $45-60$ \\
Aged & $58-72$ \\
Old & $70-86$ \\
Very old & $>80$ \\
\hline
\end{tabular}

TABle 4

\begin{tabular}{lc}
\hline \multicolumn{2}{c}{ Body mass index $\left(\mathrm{kg} / \mathrm{m}^{2}\right)$} \\
\hline Low (underweight) & $10-18$ \\
Medium (normal weight) & $15-26$ \\
Above medium (overweight) & $25-34$ \\
High (obese) & $32-40$ \\
Very high (severe obese) & $38-46$ \\
Very very high (super obese) & $44-50$ \\
\hline
\end{tabular}

$$
\begin{gathered}
\mu_{\text {old }}= \begin{cases}0 & x<70 \\
\frac{x-70}{4} & 70 \leq x \leq 74 \\
\frac{86-x}{8} & 74 \leq x \leq 78 \\
0 & x \geq 86,\end{cases} \\
\mu_{\text {very old }}= \begin{cases}0 & x \leq 80 \\
\left(\frac{x-80}{7}\right)^{2} & 80 \leq x<87 \\
1-\left(\frac{x-87}{8}\right)^{2} & 87 \leq x<95 \\
1 & x \geq 95 .\end{cases}
\end{gathered}
$$

2.1.4. BMI. Body mass index is defined as the individual's body weight divided by square of his or her height. This input field is classified into four fuzzy sets. The fuzzy sets with their range are shown in Table 4 . Consider

$$
\begin{gathered}
\mu_{\text {low }}= \begin{cases}1 & x \leq 10 \\
\left(\frac{x-10}{4}\right)^{2} & 10 \leq x<14 \\
1-\left(\frac{x-14}{4}\right)^{2} & 14 \leq x<18 \\
0 & x \geq 18,\end{cases} \\
\mu_{\text {medium }}= \begin{cases}0 & x \leq 15 \\
\frac{x-15}{3} & 15<x \leq 18 \\
\frac{26-x}{2} & 18<x \leq 24 \\
0 & 24<x \leq 26\end{cases}
\end{gathered}
$$


TABLE 5

\begin{tabular}{lc}
\hline \multicolumn{2}{c}{ Heart rate (beats/min) } \\
\hline Low & $50-65$ \\
Normal & $60-80$ \\
High & $78-110$ \\
Very high & $105-125$ \\
\hline
\end{tabular}

TABLE 6

\begin{tabular}{lc}
\hline & Triglyceride $(\mathrm{mg} / \mathrm{dL})$ \\
\hline Normal & $<150$ \\
A little bit high & $150-200$ \\
High & $200-500$ \\
Very high & $\geq 500$ \\
\hline
\end{tabular}

$$
\mu_{\text {very very high }}= \begin{cases}0 & x \leq 44 \\ \left(\frac{x-44}{3}\right)^{2} & 44 \leq x<47 \\ 1-\left(\frac{x-47}{3}\right)^{2} & 47 \leq x<50 \\ 1 & x \geq 50 .\end{cases}
$$

(6)

2.1.5. Heart Rate. This input field is classified into four linguistic variables.The fuzzy sets with their range are shown in Table 5. Consider

$$
\mu_{\mathrm{low}}= \begin{cases}1 & x \leq 50 \\ \left(\frac{x-50}{6}\right)^{2} & 50 \leq x<56 \\ 1-\left(\frac{x-56}{9}\right)^{2} & 56 \leq x<65 \\ 0 & x \geq 65\end{cases}
$$

2.1.6. Triglyceride. Triglycerides have been identified to play a major role in heart disease and hypertension. This input field is classified into four fuzzy sets. The fuzzy sets with their range are shown in Table 6. Consider

$$
\mu_{\text {normal }}= \begin{cases}1 & x \leq 0 \\ \left(\frac{x-75}{75}\right)^{2} & 0 \leq x<75 \\ 1-\left(\frac{x-75}{75}\right)^{2} & 75 \leq x<150 \\ 0 & x \geq 150,\end{cases}
$$

$$
\mu_{\text {a little bit high }}= \begin{cases}0 & x \leq 150 \\ \frac{x-150}{15} & 150<x \leq 165 \\ 1 & 165 \leq x \leq 185 \\ \frac{200-x}{15} & 185<x \leq 200 \\ 0 & x \geq 200,\end{cases}
$$


TABle 7

\begin{tabular}{lcc}
\hline & Exercise (in Min) & \\
\hline Low effective & $5-30$ \\
medium effective & $30-60$ \\
High effective & $60-100$ \\
Very high effective & $90-120$ \\
\hline
\end{tabular}

$$
\begin{gathered}
\mu_{\text {high }}= \begin{cases}0 & x \leq 200 \\
\frac{x-200}{100} & 200<x \leq 300 \\
\frac{500-x}{100} & 300 \leq x \leq 400 \\
0 & 400<x \leq 500\end{cases} \\
\mu_{\text {very high }}= \begin{cases}0 & x \leq 500, \\
\left(\frac{x-500}{100}\right)^{2} & 500 \leq x<600 \\
1-\left(\frac{x-600}{100}\right)^{2} & 600 \leq x<700 \\
1 & x \geq 700 .\end{cases}
\end{gathered}
$$

2.1.7. Physical Exercise. This input field is classified into four fuzzy sets. The fuzzy sets with their range are shown in Table 7. If a person is not doing exercise, then input value is zero. Consider

$$
\begin{gathered}
\mu_{\text {low }}= \begin{cases}1 & x \leq 5 \\
\left(\frac{x-5}{12}\right)^{2} & 5 \leq x<17 \\
1-\left(\frac{x-17}{13}\right)^{2} & 17 \leq x<30 \\
0 & x \geq 30,\end{cases} \\
\mu_{\text {medium }}= \begin{cases}\frac{x-30}{10} & 30<30 \\
\frac{1}{60-x} & 40 \leq x \leq 50 \\
\frac{60}{10} & 50<x \leq 60 \\
0 & x \geq 60,\end{cases} \\
\mu_{\text {high }}= \begin{cases}0 & x \leq 60 \\
\frac{x-60}{10} & 60<x \leq 70 \\
1 & 70 \leq x \leq 90 \\
\frac{100-x}{10} & 90<x \leq 100 \\
0 & x \geq 100,\end{cases}
\end{gathered}
$$

Table 8

\begin{tabular}{lc}
\hline \multicolumn{2}{c}{ Smoking (per day) } \\
\hline Low smoker & $5-10$ cigarettes \\
Medium smoker & $8-20$ cigarettes \\
High smoker & $18-30$ cigarettes \\
Very high smoker & $28-35$ cigarettes \\
\hline
\end{tabular}

$$
\mu_{\text {very high }}= \begin{cases}0 & x \leq 90 \\ \left(\frac{x-90}{15}\right)^{2} & 90 \leq x<105 \\ 1-\left(\frac{x-105}{15}\right)^{2} & 105 \leq x<120 \\ 1 & x \geq 120\end{cases}
$$

2.1.8. Smoking. This input field is classified into four fuzzy sets. The fuzzy sets with their range are shown in Table 8. If person is not smoking, then input value is zero. Consider

$$
\begin{aligned}
& \mu_{\text {low }}= \begin{cases}1 & x \leq 5 \\
\left(\frac{x-5}{2}\right)^{2} & 5 \leq x<7 \\
1-\left(\frac{x-7}{3}\right)^{2} & 7 \leq x<10 \\
0 & x \geq 10\end{cases} \\
& \mu_{\text {medium }}= \begin{cases}0 & x \leq 8 \\
\frac{x-8}{6} & 8<x \leq 14 \\
\frac{20-x}{6} & 14<x \leq 20 \\
0 & x \geq 20\end{cases} \\
& \mu_{\text {high }}= \begin{cases}0 & x \leq 18 \\
\frac{x-18}{6} & 18<x \leq 24 \\
\frac{30-x}{6} & 24<x \leq 30 \\
0 & x \geq 30\end{cases} \\
& \mu_{\text {very high }}= \begin{cases}0 & x \leq 28 \\
\left(\frac{x-28}{4}\right)^{2} & 28 \leq x<32 \\
1-\left(\frac{x-32}{3}\right)^{2} & 32 \leq x<35 \\
1 & x \geq 35 .\end{cases}
\end{aligned}
$$

2.2. Output Variable. The output contains risk grade of hypertension which is classified in five linguistic variables, very low, low, moderate, high, and very high. The output optimal alternatives indicate patient's present grade of hypertension. These optimal alternatives have been graphically shown in the form of Sugeno's spikes. 


\section{Result}

Now we have developed various linguistic strings to represent the state of the patient using the input variables such as age, LDL, HDL, SBP, DBP, triglyceride, BMI, HR, exercise, and smoking. Some of the linguistic strings are given as follows.

$J_{1}=$ Young $_{\text {Age }}$ Normal $_{\mathrm{LDL}}$ Normal $_{\mathrm{HDL}}$ Desirable $_{\mathrm{SBP}}$ Desirable $_{\mathrm{DBP}}$ Normal $_{\text {Triglyceride }} \quad \mathrm{M}_{\mathrm{BMI}}$ Normal $_{\mathrm{HR}}$ $\mathrm{No}_{\text {Excercise }} \mathrm{No}_{\text {Smoking }}$

$J_{2}=$ Young $_{\text {Age }}$ Normal $_{\mathrm{LDL}}$ Normal $_{\mathrm{HDL}}$ Desirable $_{\mathrm{SBP}}$ Desirable $_{\mathrm{DBP}}$ Normal $_{\text {Triglyceride }} \quad \mathrm{M}_{\mathrm{BMI}}$ Normal $_{\mathrm{HR}}$ Low $_{\text {Excercise }}$ Low $_{\text {Smoking }}$

$J_{3}=$ Young $_{\text {Age }}$ Normal $_{\mathrm{LDL}}$ Normal $_{\mathrm{HDL}}$ Desirable $_{\mathrm{SBP}}$ Desirable $_{\mathrm{DBP}}$ Normal $_{\text {Triglyceride }} \quad \mathrm{M}_{\mathrm{BMI}}$ Normal $_{\mathrm{HR}}$ Medium $_{\text {Excercise }}$ Low $_{\text {Smoking }}$

$J_{400}=$ Young $_{\text {Age }}$ Normal $_{\text {LDL }}$ Normal $_{\mathrm{HDL}}$ Normal $_{\mathrm{SBP}}$ Normal $_{\text {DBP }}$ Normal $_{\text {Triglyceride }} \mathrm{AM}_{\mathrm{BMI}} \quad$ Normal $_{\mathrm{HR}}$ Medium $_{\text {Excercise }}$ Low $_{\text {Smoking }}$

$J_{401}=$ Young $_{\text {Age }}$ Normal $_{\mathrm{LDL}}$ Normal $_{\mathrm{HDL}}$ Desirable $_{\mathrm{SBP}}$ Desirable $_{\mathrm{DBP}}$ Normal $_{\text {Triglyceride }} \mathrm{AM}_{\mathrm{BMI}} \quad$ High $_{\mathrm{HR}}$ Medium $_{\text {Excercise }}$ Med $_{\text {Smoking }}$

$J_{2500}=$ Adult $_{\text {Age }}$ Normal $_{\mathrm{LDL}}$ Normal $_{\mathrm{HDL}}$ Desirable $_{\mathrm{SBP}}$ ADesirable $_{\mathrm{DBP}}$ Normal $_{\text {Triglyceride }} \mathrm{M}_{\mathrm{BMI}} \quad$ Normal $_{\mathrm{HR}}$ $\mathrm{No}_{\text {Excercise }} \mathrm{No}_{\text {Smoking }}$

$J_{6500}=$ Adult $_{\text {Age }}$ High $_{\mathrm{LDL}}$ High $_{\mathrm{HDL}}$ Desirable $_{\mathrm{SBP}}$ Moderate $_{\mathrm{DBP}}$ Normal $_{\text {Triglyceride }} \mathrm{AM}_{\mathrm{BMI}}$ High $_{\mathrm{HR}} \mathrm{LOW}_{\text {Excercise }}$ Low $_{\text {Smoking }}$

$J_{10001}=$ Adult $_{\text {Age }}$ High $_{\mathrm{LDL}}$ High $_{\mathrm{HDL}}$ Desirable $_{\mathrm{SBP}}$ Moderate $_{\mathrm{DBP}}$ Normal $_{\text {Triglyceride }} \mathrm{AM}_{\mathrm{BMI}}$ High $_{\mathrm{HR}}$ Low $_{\text {Excercise }}$ Medium $_{\text {Smoking }}$

$J_{17009}=$ Midaged $_{\text {Age }}$ Normal $_{\text {LDL }}$ Normal $_{\text {HDL }}$ Desirable $_{\mathrm{SBP}}$ Desirable DBP Normal $_{\text {Triglyceride }} \mathrm{M}_{\mathrm{BMI}}$ Nor$\mathrm{mal}_{\mathrm{HR}} \mathrm{No}_{\text {Excercise }} \mathrm{No}_{\text {Smoking }}$

$J_{17010}=$ Midaged $_{\text {Age }}$ Normal $_{\text {LDL }}$ Normal $_{\text {HDL }}$ Desirable $_{\text {SBP }}$ Desirable $_{\text {DBP }}$ Normal $_{\text {Triglyceride }} \mathrm{M}_{\mathrm{BMI}}$ High $_{\mathrm{HR}}$ $\mathrm{No}_{\text {Excercise }}$ Low $_{\text {Smoking }}$
$J_{200080}=$ Aged $_{\text {Age }}$ Normal $_{\text {LDL }}$ Normal $_{\text {HDL }}$ Desirable $_{\mathrm{SBP}}$ ADesirable $_{\text {DBP }}$ Normal $_{\text {Triglyceride }} \mathrm{M}_{\mathrm{BMI}} \quad$ Normal $_{\mathrm{HR}}$ $\mathrm{No}_{\text {Excercise }} \mathrm{No}_{\text {Smoking }}$

$J_{405001}=$ Aged $_{\text {Age }}$ High $_{\mathrm{LDL}}$ High $_{\mathrm{HDL}}$ Moderate $_{\mathrm{SBP}}$ Moderate $_{\mathrm{DBP}}$ Normal $_{\text {Triglyceride }} \mathrm{VH}_{\mathrm{BMI}} \quad \mathrm{VH}_{\mathrm{HR}}$ Low $_{\text {Excercise }}$ Medium $_{\text {Smoking }}$

$J_{966080}=$ Aged $_{\text {Age }}$ VeryHigh $_{\text {LDL }}$ NVeryhigh $_{\mathrm{HDL}}$ Veryhigh $_{\mathrm{SBP}}$ Veryhigh $_{\mathrm{DBP}}$ Veryhigh $_{\text {Triglyceride }} \mathrm{VH}_{\mathrm{BMI}}$ Normal $_{\mathrm{HR}}$ Low $_{\text {Excercise }}$ high $_{\text {Smoking }}$

$J_{1200200}=$ Old $_{\text {Age }}$ High $_{\text {LDL }}$ Normal $_{\mathrm{HDL}}$ AboveDesirable $_{\mathrm{SBP}}$ Moderate $\mathrm{DBP}$ Normal $_{\text {Triglyceride }} \mathrm{AM}_{\mathrm{BMI}}$ Normal $_{\text {HR }}$ Low $_{\text {Excercise }} \mathrm{No}_{\text {Smoking }}$

$J_{2344569}=\operatorname{Old}_{\text {Age }}$ VeryHigh $_{\text {LDL }}$ NVeryhigh $_{\mathrm{HDL}}$ Veryhigh $_{\mathrm{SBP}}$ high $_{\mathrm{DBP}}$ Veryhigh $_{\text {Triglyceride }} \mathrm{AM}_{\mathrm{BMI}} \mathrm{High}_{\mathrm{HR}}$ $\mathrm{No}_{\text {Excercise }}$ Very high Smoking

$J_{5676880}=$ VeryOld $_{\text {Age }}$ Veryhigh $_{\text {LDL }}$ Normal $_{\text {HDL }}$ Moderate $_{\mathrm{SBP}} \mathrm{ADesirable} \mathrm{DBP}_{\mathrm{D}}$ Normal $_{\text {Triglyceride }} \mathrm{AM}_{\mathrm{BMI}}$ Normal $_{\text {HR }}$ Low $_{\text {Excercise }}$ Low $_{\text {Smoking }}$

$J_{7566780}=$ Veryold $_{\text {Age }}$ High $_{\text {LDL }}$ High $_{\mathrm{HDL}}$ Veryhigh $_{\mathrm{SBP}}$ Veryhigh $_{\mathrm{DBP}}$ Veryhigh $_{\text {Triglyceride }} \quad \mathrm{High}_{\mathrm{BMI}} \mathrm{VH}_{\mathrm{HR}}$ $\mathrm{No}_{\text {Excercise }}$ Veryhigh $_{\text {Smoking }}$

$J_{8545200}=$ Veryold $_{\text {Age }}$ VeryHigh $_{\text {LDL }}$ Veryhigh $_{\mathrm{HDL}}$ Veryhigh $_{\mathrm{SBP}}$ High $_{\mathrm{DBP}}$ V.high $_{\text {Triglyceride }} \mathrm{VH}_{\mathrm{BMI}} \mathrm{High}_{\mathrm{HR}}$ $\mathrm{No}_{\text {Excercise }}$ high $\mathrm{Smoking}_{\text {Sm }}$

$J_{9031678}=$ Veryold $_{\text {Age }}$ VeryHigh $_{\text {LDL }}$ Veryhigh $_{\mathrm{HDL}}$ Veryhigh $_{\mathrm{SBP}}$ Veryhigh $_{\mathrm{DBP}}$ Veryhigh $_{\text {Triglyceride }} \mathrm{VH}_{\mathrm{BMI}}$ $\mathrm{VH}_{\mathrm{HR}}$ Low $_{\text {Excercise }}$ Very high Smoking

$J_{9031680}=$ Veryold $_{\text {Age }}$ VeryHigh $_{\text {LDL }}$ Veryhigh $_{\mathrm{HDL}}$ Veryhigh $_{\mathrm{SBP}}$ Veryhigh $_{\mathrm{DBP}}$ Veryhigh $_{\text {Triglyceride }}$ $\mathrm{MVVH}_{\mathrm{BMI}} \mathrm{VH}_{\mathrm{HR}} \mathrm{No}_{\text {Excercise }}$ Very high $\mathrm{S}_{\text {Smoking }}$.

On the basis of our proposed technique to investigate the health status of a person whose medical data is available, three different cases have been discussed as follows.

Case 1. The input variables of first patient are

(1) Age $=\{(0$, young $),(0.8$, adult $),(0$, midaged $),(0$, aged $)$, $(0$, old $),(0$, very old $)\}$.

(2) $\mathrm{LDL}=\{(0.71$, normal $),(0$, above normal $)$, ( 0 , borderline high), (0, high), (0, very high) . 
(3) $\mathrm{HDL}=\{(0$, very high $),(0$, high $),(0$, nearly normal $)$, $(1$, normal $)\}$.

(4) $\mathrm{SBP}=\{(0$, desirable $),(0.8$, above desirable $),(0$, moderate), (0, above moderate), ( 0 , little high), $(0$, high $)$, ( 0 , very high $)\}$.

(5) $\mathrm{DBP}=\{(0$, desirable $),(1$, above desirable $),(0$, moderate), (0, above moderate), ( 0 , little high $),(0$, high $),(0$, very high)\}.

(6) Triglyceride $=\{(0.72$, normal $),(0$, a little bit high $),(0$, high), (0, very high $)\}$.

(7) $\mathrm{BMI}=\{(0$, low $),(0.99$, medium $),(0$, above medium $)$, $(0$, high $),(0$, very high $),(0$, very very high $)\}$.

(8) $\operatorname{HR}=\{(0$, low $),(0.86$, normal $),(0$, high $),(0$, very high)\}.

(9) Exercise $=\{(1$, low $),(0$, medium $),(0$, high $),(0$, very high)\}.

(10) Smoking $=\{(1$, low $),(0$, medium $),(0$, high $),(0$, very high)\}.

This is the fuzzy set which represents the state of concerned patient:

$$
X=\left\{\left(0.71, J_{A, N, N, A D, A D, N, M, N, L, L}\right)\right\} .
$$

The utility matrix $U$ of order $5 x 9031680$ by using the fuzzy rule base designed is as follows:

$$
U=\left(\begin{array}{rrrrrrrr}
98 & 95 & \cdots & 10 & \cdots & 10 & \cdots & 05 \\
68 & 80 & \cdots & 20 & \cdots & 30 & \cdots & 10 \\
30 & 25 & \cdots & 45 & \cdots & 62 & \cdots & 40 \\
20 & 20 & \cdots & 92 & \cdots & 90 & \cdots & 86 \\
10 & 10 & \cdots & 55 & \cdots & 20 & \cdots & 95
\end{array}\right)
$$

The five fuzzy utilities are

(1) $U_{1}=\{(0.71,98)\}$,

(2) $U_{2}=\{(0.71,68)\}$,

(3) $U_{3}=\{(0.71,30)\}$,

(4) $U_{4}=\{(0.71,20)\}$,

(5) $U_{5}=\{(0.71,10)\}$.

The maximum sets corresponding to each alternative are

(1) $U_{1 m}=\{(1,98)\}$,

(2) $U_{2 m}=\{(0.69,68)\}$,

(3) $U_{3 m}=\{(0.30,30)\}$,

(4) $U_{4 m}=\{(0.20,20)\}$,

(5) $U_{5 m}=\{(0.10,10)\}$.

Now, the optimal fuzzy utilities using fuzzy utilities and maximizing sets are

(1) $U_{1 o}=\{(0.71,98)\}$,

(2) $U_{2 o}=\{(0.69,68)\}$,

(3) $U_{30}=\{(0.30,30)\}$,

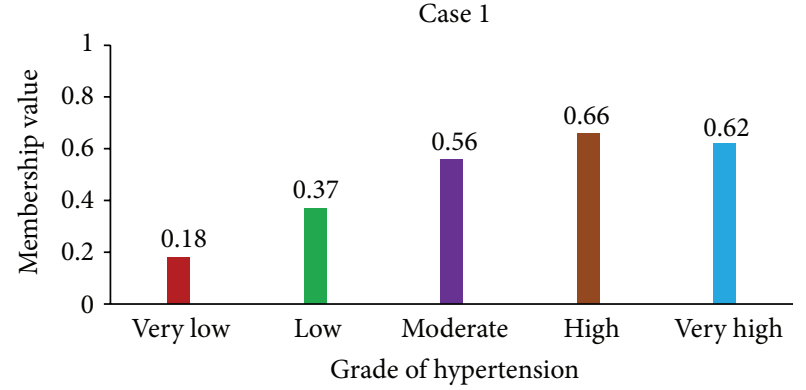

FIgURE 1: View of Sugeno's spikes.

(4) $U_{4 o}=\{(0.20,20)\}$,

(5) $U_{50}=\{(0.10,10)\}$.

Using these utilities, the optimal alternatives are given by $A_{o}=\{(0.71$, very low $),(0.69$, low $),(0.30$, moderate $)$,

$$
(0.20 \text {, high }) \text {, and }(0.10 \text {, very high })\} \text {. }
$$

This optimal alternative indicates that the patient presently is in very low grade of hypertension. The optimal alternatives have been graphically shown in Figure 1 in the form of Sugeno's spikes.

Case 2. The input variables of the second patient are as follows.

(1) Age $=\{(0$, young $),(0$, adult $),(1$, midaged $),(0$, aged $)$, $(0$, old $),(0$, very old $)\}$.

(2) $\mathrm{LDL}=\{(1$, normal $),(0$, above normal $),(0$, borderline high), (0, high), (0, very high) $\}$.

(3) $\mathrm{HDL}=\{(0$, very high $),(0.6$, high $),(0$, nearly normal $)$, $(0$, normal $)\}$.

(4) $\mathrm{SBP}=\{(0$, desirable $),(0.8$, above desirable $),(0$, moderate), (0, above moderate), ( 0 , little high), ( 0 , high), (0, very high)\}.

(5) $\mathrm{DBP}=\{(0$, desirable $),(0.66$, above desirable $),(0$, moderate), (0, above moderate), (0, little high), $(0$, high), (0, very high)\}.

(6) Triglyceride $=\{(0.66$, normal $),(0$, a little bit high $),(0$, high), (0, very high) $\}$.

(7) $\mathrm{BMI}=\{(0$, low $),(0$, medium $),(0.86$, above medium $)$, $(0$, high $),(0$, very high $),(0$, very very high $)\}$.

(8) $\mathrm{HR}=\{(0$, low $),(0.76$, normal $),(0$, high $),(0$, very high)\}.

(9) Exercise $=\{(0$, low $),(0.79$, medium $),(0$, high $),(0$, very high) $\}$.

(10) Smoking $=\{(1$, low $),(0$, medium $),(0$, high $),(0$, very high)\}.

This is the fuzzy set which represents the state of concerned patient:

$$
X=\left\{\left(0.6, J_{M A, N, H, A D, A D, N, A M, N, M, L}\right)\right\} .
$$




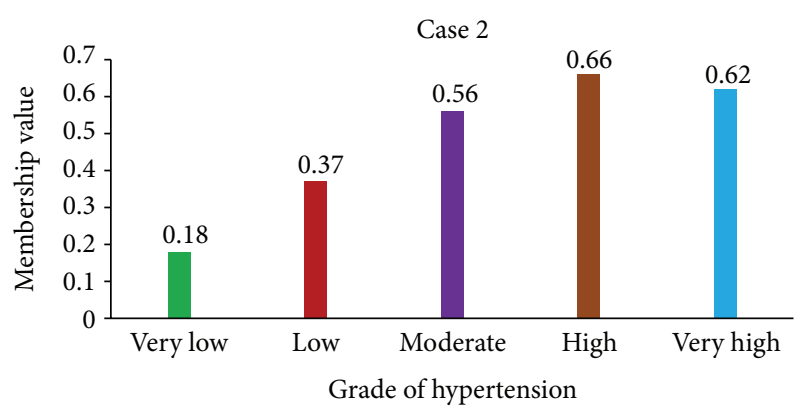

FIgURE 2: View of Sugeno's spikes.

The utility matrix is the same as in Case 1.

The five fuzzy utilities are

(1) $U_{1}=\{(0.6,55)\}$,

(2) $U_{2}=\{(0.6,92)\}$,

(3) $U_{3}=\{(0.6,40)\}$,

(4) $U_{4}=\{(0.6,24)\}$,

(5) $U_{5}=\{(0.6,10)\}$.

The maximum sets corresponding to each alternative are

(1) $U_{1 m}=\{(0.59,55)\}$,

(2) $U_{2 m}=\{(1,92)\}$,

(3) $U_{3 m}=\{(0.43,35)\}$,

(4) $U_{4 m}=\{(0.26,24)\}$,

(5) $U_{5 m}=\{(0.10,10)\}$.

Now, the optimal fuzzy utilities using fuzzy utilities and maximizing sets are

(1) $U_{1 o}=\{(0.59,55)\}$,

(2) $U_{2 o}=\{(0.6,92)\}$,

(3) $U_{30}=\{(0.43,35)\}$,

(4) $U_{4 o}=\{(0.26,24)\}$,

(5) $U_{5 o}=\{(0.10,10)\}$.

Using these utilities, the optimal alternatives are given by $A_{o}=\{(0.59$, very low $),(0.60$, low $),(0.43$, moderate $)$,

$(0.26$, high $)$, and $(0.10$, very high $)\}$.

This optimal alternative indicates that the patient presently is in low grade of hypertension. The optimal alternatives have been graphically shown in Figure 2 in the form of Sugeno's spikes.

Case 3. The input variables of the third patient are as follows.

(1) Age $=\{(0$, young $),(0$, adult $),(0$, midaged $),(0$, aged $)$, $(0.75$, old $),(0$, very old $)\}$.

(2) $\mathrm{LDL}=\{(0$, normal $),(0$, above normal $),(0$, borderline high), (0.8, high), (0, very high) $\}$.
(3) $\mathrm{HDL}=\{(0$, very high $),(1$, high $),(0$, nearly normal $)$, $(0$, normal $)\}$.

(4) $\mathrm{SBP}=\{(0$, desirable $),(0$, above desirable $),(0.8$, moderate), (0, above moderate), (0, little high), (0, high), $(0$, very high $)\}$.

(5) $\mathrm{DBP}=\{(0$, desirable $),(1$, above desirable $),(0$, moderate), (0, above moderate), (0, little high), (0, high), ( 0 , very high)\}.

(6) Triglyceride $=\{(1$, normal $),(0.66$, a little bit high $),(0$, high), (0, very high)\}.

(7) $\mathrm{BMI}=\{(0$, low $),(0$, medium $),(0$, above medium $)$, $(0.78$, high $),(0$, very high $),(0$, very very high $)\}$.

(8) $\mathrm{HR}=\{(0$, low $),(0$, normal $),(0.96$, high $)$, (0, very high)\}.

(9) Exercise $=\{(0$, low $),(1$, medium $),(0$, high $)$, (0, very high)\}.

(10) Smoking $=\{(1$, low $),(0$, medium $),(0$, high $),(0$, very high)\}.

This is the fuzzy set which represents the state of concerned patient:

$$
X=\left\{\left(0.66, J_{O, H, H, M, A D, L H, H, H, M, L}\right)\right\} .
$$

The utility matrix $U$ is the same as in Case 1 .

The five fuzzy utilities are

(1) $U_{1}=\{(0.66,15)\}$,

(2) $U_{2}=\{(0.66,30)\}$,

(3) $U_{3}=\{(0.66,45)\}$,

(4) $U_{4}=\{(0.66,80)\}$,

(5) $U_{5}=\{(0.66,50)\}$.

The maximum sets corresponding to each alternative are

(1) $U_{1 m}=\{(0.187,15)\}$,

(2) $U_{2 m}=\{(0.375,30)\}$,

(3) $U_{3 m}=\{(0.562,45)\}$,

(4) $U_{4 m}=\{(1,80)\}$,

(5) $U_{5 m}=\{(0.62,50)\}$.

Now, the optimal fuzzy utilities using fuzzy utilities and maximizing sets are

(1) $U_{1 o}=\{(0.187,15)\}$,

(2) $U_{2 o}=\{(0.375,30)\}$,

(3) $U_{3 o}=\{(0.562,45)\}$,

(4) $U_{4 o}=\{(0.66,80)\}$,

(5) $U_{5 o}=\{(0.62,50)\}$.

Using these utilities, the optimal alternatives are given by $A_{o}=\{(0.187$, very low $),(0.375$, low $),(0.562$, moderate $)$, $(0.66$, high $)$, and $(0.62$, very high $)\}$. 


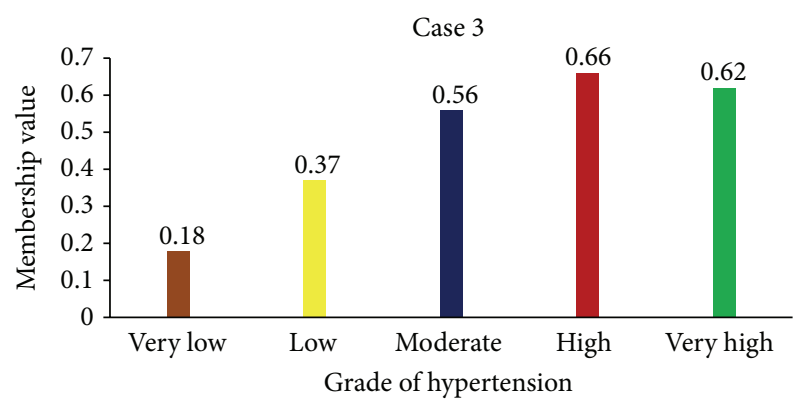

Figure 3: View of Sugeno's spikes.

This optimal alternative indicates that the patient is presently in high grade of hypertension.

The optimal alternatives have been graphically shown in the form of Sugeno's spikes in Figure 3.

\section{Conclusion}

The present research paper confirms that the soft computing diagnostic system can represent the expert's thinking in an efficient manner to handle complex cases. The design and development of soft computing risk assessment system on the basis of the proposed technique will assist medical experts to measure grade classification of hypertension efficiently.

\section{References}

[1] X. Y. Djam and Y. H. Kimbi, "Fuzzy expert system for the management of hypertension," The Pacific Journal of Science and Technology, vol. 12, no. 1, p. 390, 2011.

[2] X. Y. Djam and Y. H. Kimb, "A medical diagnostic support system for the management of Hypertension (MEDDIAG)," Journal of Medical and Applied Biosciences, vol. 3, pp. 41-55, 2011.

[3] R. Jain, "Decision making in the presence of fuzzy variables," IEEE Transactions on Systems, Man and Cybernetics, vol. 6, no. 10, pp. 698-703, 1976.

[4] R. Poli, S. Cagnoni, R. Livi et al., "A neural network expert systemfor diagnosing and treating hypertension," Computer, vol. 24, no. 3, pp. 64-71, 1991.

[5] R. Degani, "Computerized electrocardiogram diagnosis: fuzzy approach," Methods of Information in Medicine, vol. 31, no. 4, pp. 225-233, 1992.

[6] S. Charbonnier, S. Galichet, G. Mauris, and J. P. Siché, "Statistical and fuzzy models of ambulatory systolic blood pressure for hypertension diagnosis," IEEE Transactions on Instrumentation and Measurement, vol. 49, no. 5, pp. 998-1003, 2000.

[7] R. K. Jena, M. M. Aqel, P. Srivastava, and P. K. Mahanti, "Soft computing methodologies in bioinformatics," European Journal of Scientific Research, vol. 26, no. 2, pp. 189-203, 2009.

[8] D. Pandey, M. Vaishali, and S. Pankaj, "Rule-based system for cardiac analysis," National Academy Science Letters, vol. 29, no. 7-8, pp. 299-309, 2006.

[9] N. Allahverdi, S. Torun, and I. Saritas, "Design of a fuzzy expert system for determination of coronary heart disease risk," in International Conference on Computer Systems and Technologies (CompSysTech '07), June 2007.
[10] N. Nalayini and R. S. D. Wahidabanu, "Design methodology of a controller to forecast the uncertain cardiac arrest using fuzzy logic approach," in Proceedings of the International Conference Intelligent Systems and Controls, pp. 46-52, Karpagam College of Engineering, Coimbatore, India, 2008.

[11] P. Srivastava and A. Srivastava, "A soft computing approach for cardiac analysis," Journal of Basic and Applied Scientific Research, vol. 2, no. 1, pp. 376-385, 2012.

[12] P. Srivastava and N. Sharma, "Soft computing criterion for ECG beat classification and cardiac analysis," communicated, 2012. 


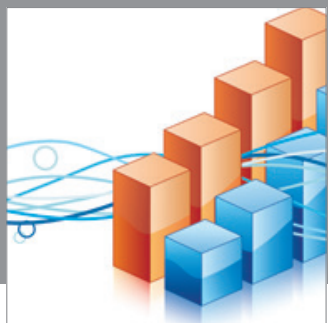

Advances in

Operations Research

mansans

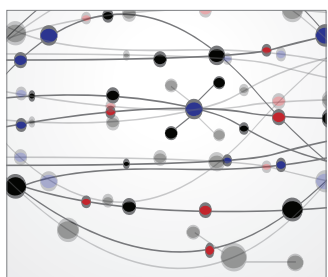

The Scientific World Journal
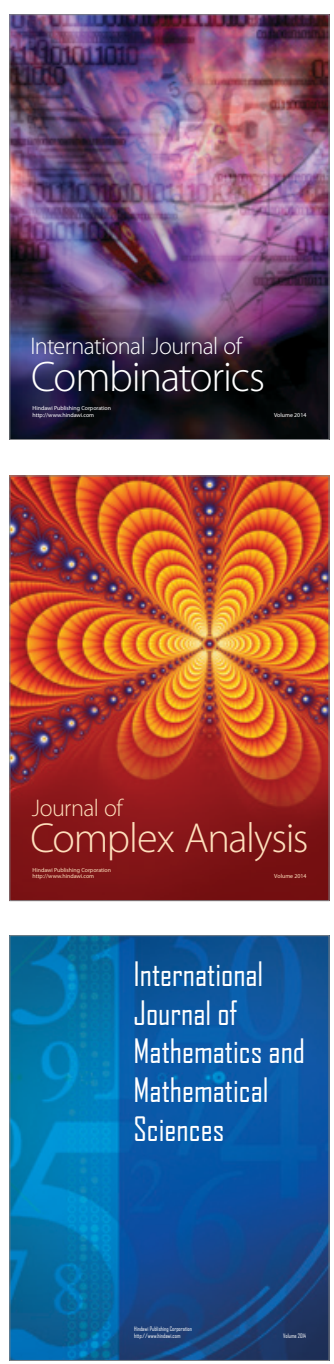
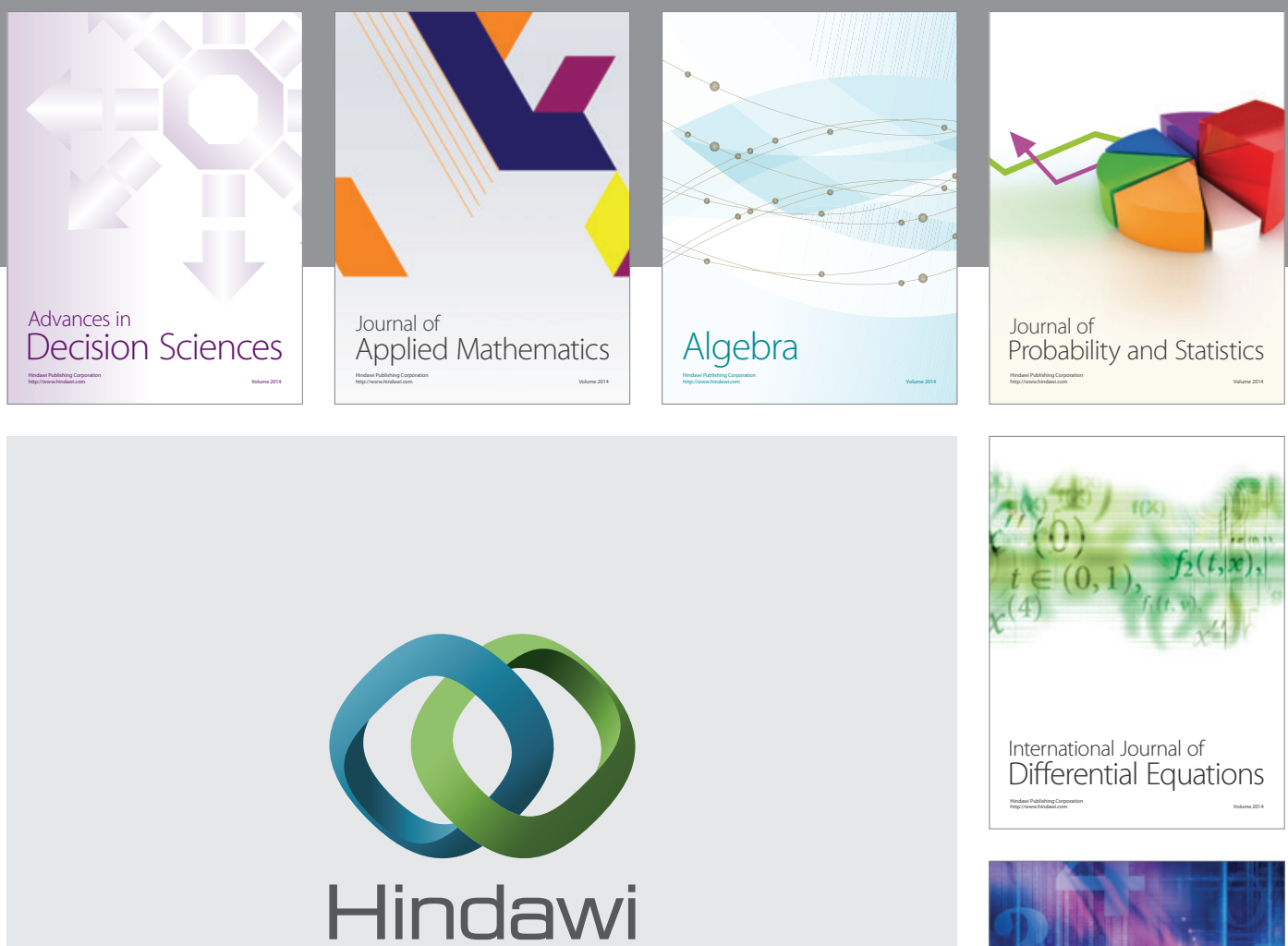

Submit your manuscripts at http://www.hindawi.com
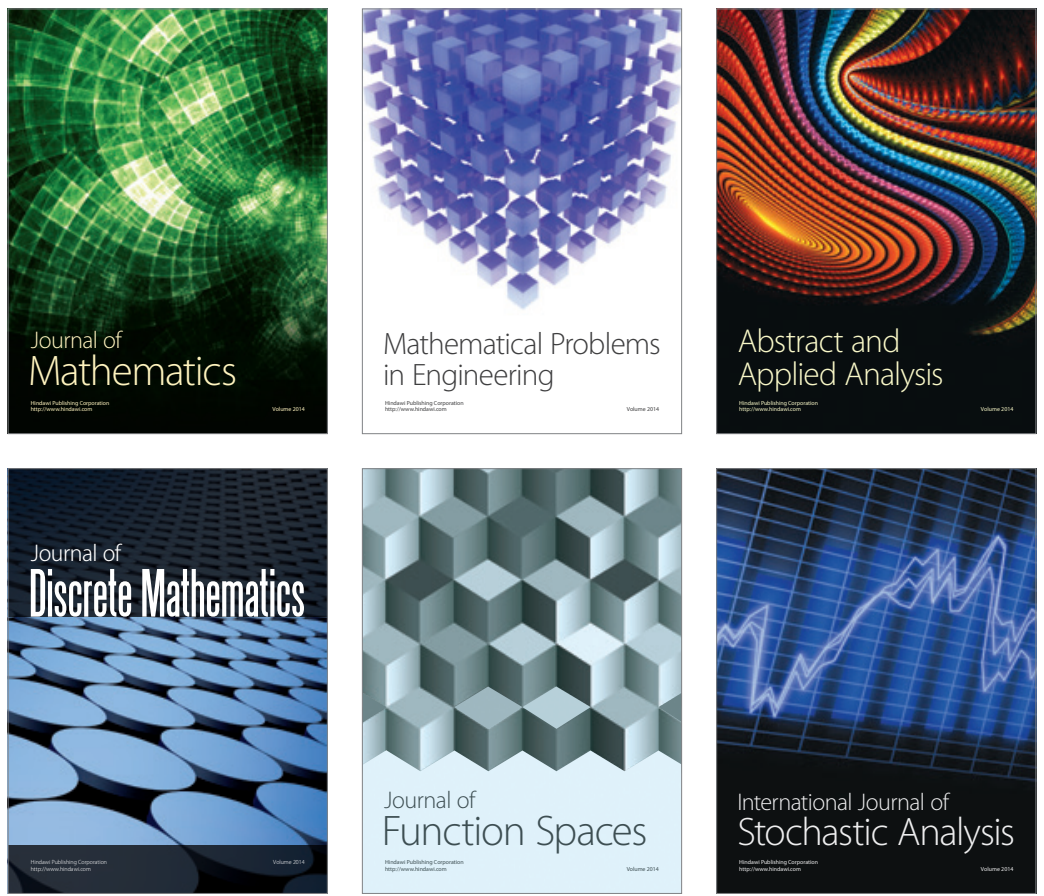

Journal of

Function Spaces

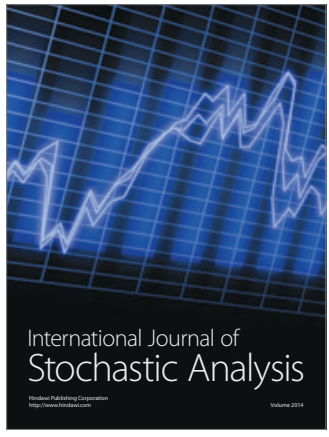

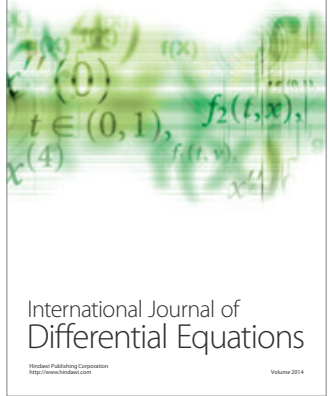
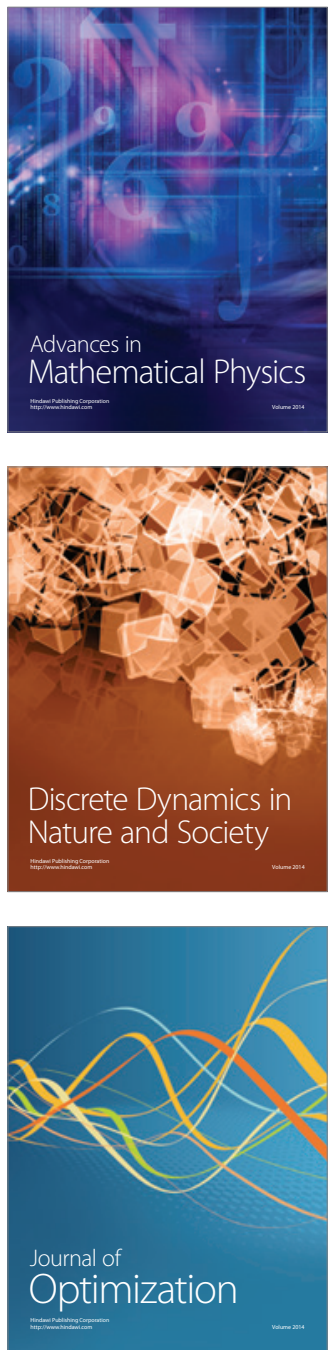